\title{
Unplanned readmissions and survival following brain tumor surgery
}

\author{
Holly Dickinson, BS, Christine Carico, BS, Miriam Nuño, PhD, Debraj Mukherjee, MD, MPH, \\ Alicia Ortega, BA, Keith L. Black, MD, and Chirag G. Patil, MD, MS
}

Center for Neurosurgical Outcomes Research, Maxine Dunitz Neurosurgical Institute, Department of Neurosurgery, Cedars-Sinai Medical Center, Los Angeles, California

\begin{abstract}
OBJECT Research on readmissions has been influenced by efforts to reduce hospital cost and avoid penalties stipulated by the Centers for Medicare and Medicaid Services. Less emphasis has been placed on understanding these readmissions and their impact on patient outcomes. This study 1) delineates reasons for readmission, 2) explores factors associated with readmissions, and 3) describes their impact on the survival of glioblastoma patients.

METHODS The authors conducted a retrospective review of 362 cases involving patients with glioblastoma undergoing biopsy or tumor resection at their institution between 2003 and 2011. Reasons for re-hospitalization were characterized according to whether or not they were related to surgery and considered preventable. Multivariate analyses were conducted to identify the effect of readmission on survival and determine factors associated with re-hospitalizations.

RESULTS Twenty-seven (7.5\%) of 362 patients experienced unplanned readmissions within 30 days of surgery. Six patients (22.2\%) were readmitted by Day 7, $14(51.9 \%)$ by Day 14 , and $20(74.1 \%)$ by Day 21. Neurological, infectious, and thromboembolic complications were leading reasons for readmission, accounting for, respectively, 37.0\%, 29.6\%, and $22.2 \%$ of unplanned readmissions. Twenty-one (77.8\%) of the 27 readmissions were related to surgery and $19(70.4 \%)$ were preventable. The adjusted hazard ratio of mortality associated with a readmission was $2.03(95 \% \mathrm{Cl} 1.3-3.1)$. Higher-functioning patients (OR $0.96,95 \% \mathrm{Cl} 0.9-1.0)$ and patients discharged home (OR $0.21,95 \% \mathrm{Cl} 0.1-0.6)$ were less likely to get readmitted.
\end{abstract}

CONCLUSIONS An overwhelming fraction of documented unplanned readmissions were considered preventable and related to surgery. Patients who were readmitted to the hospital within 30 days of surgery had twice the risk of mortality compared with patients who were not readmitted.

http://thejns.org/doi/abs/10.3171/2014.8.JNS1498

KEY WORDS unplanned readmission; glioblastoma; complications; functional status; oncology

$\mathrm{I}$ $\mathrm{N}$ recent years, early hospital readmissions have emerged as a proxy measure for hospital quality of care, leading legislation regarding hospital reimbursement policy to undergo significant revision. ${ }^{38}$ As of October 1,2012, hospitals with higher standardized early readmission rates for medical conditions such as heart failure, acute myocardial infarction, and pneumonia have been challenged with payment penalties assessed by the Centers for Medicare and Medicaid Services. ${ }^{29}$ As readmission rates have already begun to affect hospital reimbursement and will continue to do so in the future, clear understanding of early readmission, as a reliable quality indicator across medical subspecialties, is critical. If early readmissions are to be used as a universal measure of quality of care, it is imperative to establish that these hospitalizations are correlated with adverse patient outcomes. Many studies in recent years have investigated early readmissions the majority of which focus on the financial and resource burden that early readmissions place upon the health care system. ${ }^{9}, 10,19,20,22,34,37$ However, fewer studies have assessed the factors driving 30-day readmissions, their association with patient outcomes, and the possible implications for quality of care. Several studies in surgical oncology have demonstrated that readmission status is associated with survival in patients with high-grade neoplasms, such as pancreatic ${ }^{28}$ and colon ${ }^{15}$ cancers. As glioblastoma is the most common and malignant of the primary brain tumors ${ }^{25}$ and surgical resection remains an important treat-

ABBREVIATIONS DVT = deep venous thrombosis; $\mathrm{HR}=$ hazard ratio; $\mathrm{KPS}=$ Karnofsky Performance Status; LOS = length of hospital stay; PE = pulmonary embolism; $\mathrm{SOI}=$ severity of illness; $\mathrm{SSI}=$ surgical site infection; UTI = urinary tract infection; VTE = venous thromboembolism.

SUBMITTED January 20, 2014. ACCEPTED August 19, 2014.

INCLUDE WHEN CITING Published online October 24, 2014; DOI: 10.3171/2014.8.JNS1498.

DISCLOSURE The authors report no conflict of interest concerning the materials or methods used in this study or the findings specified in this paper. 
ment paradigm, ${ }^{3}$ investigating readmissions in association with survival in this cohort challenged by a poor prognosis is critical.

The objectives of this study were to first establish the correlation between postsurgical 30-day readmissions and survival in glioblastoma patients and to further explore factors associated with these re-hospitalizations. In delineating reasons for readmission, we aim to elucidate potential areas of improvement in hospital care in an effort to reduce these adverse patient events.

\section{Methods}

\section{Data Source and Variables}

We performed a retrospective review of 362 cases involving newly diagnosed glioblastoma adult ( $>18$ years) patients who underwent surgery (including biopsy) at our institution for tumor resection between January 2003 and December 2011. Patient characteristics such as age, sex, race, extent of resection, and treatment (radiation and temozolomide) were included. Tumor size, perioperative Karnofsky Performance Status (KPS), disease severity, risk of mortality, and comorbidities were among some of the factors captured for these patients. Severity of illness (SOI) is defined as the extent of organ system derangement or physiological decompensation for a patient. Classified as minor, moderate, major, or extreme, $\mathrm{SOI}$ is meant to provide a basis for evaluating hospital resource use and to establish patient care guidelines. Patients with higher SOI classifications are likely to use more health care resources and experience more extended lengths of hospital stay (LOS) than patients with lower SOI within related cohorts. Similarly, the risk of mortality provides a medical classification to estimate the likelihood of in-hospital death for a patient. Levels of mortality risk are similar to those of SOI.

Discharge disposition (home, rehabilitation, or transfer) and type of admission (routine, emergency room, or transfer) were also considered. Detailed documentation describing the reasons for readmission was examined for each patient. Missing data for race were noted in $1.1 \%$ of cases and missing data for tumor size were noted in $18.2 \%$.

\section{Outcomes of Interest}

A patient's overall survival and the occurrence of a readmission within 30 days of surgery were the main outcomes of interest. A readmission was defined as an admission lasting at least overnight under the care of a service other than the emergency service. Overall survival was calculated as the time between surgery and the date of death or most recent follow-up for censored patients. Furthermore, a detailed assessment of complications that led to these readmissions was described, and the readmissions were categorized into either preventable or non-preventable. A readmission was considered to be preventable if the chief complaint included any of the following: 1) a condition that was described and coded in the index hospitalization, 2) nosocomial surgical site infection (SSI) or fluid and electrolyte imbalances, 3) deep venous thrombosis (DVT) and pulmonary embolism (PE), and 4) postoperative CSF leaks that required repair. ${ }^{34}$ Non-preventable readmissions include those related to disease progression, such as seizures, ${ }^{1}$ side effects of treatment, and hydrocephalus. ${ }^{34}$ Each readmission was further described in terms of whether it was deemed to be related to surgery. In this cohort a readmission was presumed unrelated if it was a direct result of any of the following: 1) treatment side effects, 2) new conditions not coded at index hospitalization, (3) disease progression.

\section{Statistical Analysis}

Descriptive statistics accounted for whether patients were readmitted within 30 days of index surgery. Continuous variables were described using means and medians, while categorical variables were described with frequencies. Univariate comparisons of patient characteristics by readmission were conducted using Wilcoxon rank-sum, chi-square, and Fisher's exact tests, where appropriate. The Kaplan-Meier estimation method was used to obtain median survival times and rates. The Cox proportional hazards model was used to assess the association of readmission and survival while adjusting for multiple covariates. Multivariate logistic regression was used to determine predictors of readmission; factors associated with a readmission at the univariate level with a $p$ value of 0.25 or lower were considered in the model. A p value less than or equal to 0.05 was considered statistically significant. All statistical analyses were conducted in SAS 9.2 (SAS Institute).

\section{Results \\ Demographics}

Our cohort had a median age of 63 years, with a male preponderance among both readmitted (59.3\%) and nonreadmitted $(61.2 \%)$ patients (Table 1$)$. The proportion of white patients was lower in the readmitted cohort than in the non-readmitted cohort ( $74.1 \%$ vs $86.7 \%, \mathrm{p}=0.09$ ). Readmitted patients had significantly lower perioperative KPS scores $(67.0$ vs $74.5, \mathrm{p}<0.0001)$ than non-readmitted patients. The extent of resection was similar in the 2 cohorts $(\mathrm{p}=0.51)$. Discharge disposition at the index hospitalization was strongly associated with readmission; the proportion of patients who were discharged home was substantially lower in the readmitted cohort than in the non-readmitted cohort $(33.3 \%$ vs $76.7 \%, \mathrm{p}<0.0001)$.

\section{Overall Survival and Readmission}

The median overall survival for patients who were readmitted within 30 days of surgery was significantly shorter than for non-readmitted patients (6.9 vs 16.0 months, $\mathrm{p}=0.003$ ) (Fig. 1). After adjusting for confounders in a multivariate model, we found that the occurrence of a readmission (HR 2.03, $\mathrm{p}=0.001$ ), older age (hazard ratio $[\mathrm{HR}] 1.03, \mathrm{p}<0.0001)$, and a level increase in disease severity $(\mathrm{HR} 1.21, \mathrm{p}=0.03)$ were significantly associated with higher hazard of mortality. Additionally, higher KPS score (HR 0.85, $\mathrm{p}=0.001$ ), near/gross-total resection compared with biopsy (HR 0.51, p < 0.0001), and temozolomide treatment (HR 0.44, p < 0.0001) were associated with a significant reduction in the hazard of mortality (Table 2). 
TABLE 1. Characteristics of 27 readmitted and 335 non-readmitted patients with newly diagnosed glioblastoma treated between January 2003 and December 2011*

\begin{tabular}{|c|c|c|c|}
\hline Variable & $\begin{array}{l}\text { Readmitted } \\
(n=27[7.5])\end{array}$ & $\begin{array}{c}\text { Non-Readmitted } \\
(\mathrm{n}=335[92.5])\end{array}$ & $p$ Value \\
\hline Age at diagnosis (yrs) & & & 0.62 \\
\hline Mean & $62.4 \pm 14.0$ & $61.1(13.3)$ & \\
\hline Median & 63 & 62 & \\
\hline IQR & $52-74$ & $52-70$ & \\
\hline LOS (days) & & & 0.10 \\
\hline Mean & $8.3 \pm 6.6$ & $6.3(6.3)$ & \\
\hline Median & 6 & 4 & \\
\hline IQR & $3-12$ & $3-8$ & \\
\hline Female sex & $11(40.7)$ & $130(38.8)$ & 0.84 \\
\hline Race & & & 0.11 \\
\hline White & $20(74.1)$ & $287(86.7)$ & \\
\hline Black & $3(11.1)$ & $21(6.3)$ & \\
\hline Asian & $2(7.4)$ & $16(4.8)$ & \\
\hline Other/unknown & $2(7.4)$ & $7(2.1)$ & \\
\hline Missing & $0(0)$ & $4(1.2)$ & \\
\hline Periop KPS score & & & $<0.0001$ \\
\hline Mean & $67.0 \pm 16.4$ & 74.5 (14.1) & \\
\hline Median & 70 & 80 & \\
\hline IQR & $60-80$ & $70-90$ & \\
\hline Tumor size & & & 0.92 \\
\hline$<4 \mathrm{~cm}$ & $7(33.3)$ & $108(39.3)$ & \\
\hline$>4 \mathrm{~cm}$ & $14(66.7)$ & $167(60.7)$ & \\
\hline Missing & $6(22.2)$ & $60(17.9)$ & \\
\hline EOR & & & 0.51 \\
\hline Biopsy & $11(40.7)$ & $160(47.8)$ & \\
\hline PR & $8(29.6)$ & $68(20.3)$ & \\
\hline N/GTR & $8(29.6)$ & $107(31.9)$ & \\
\hline Radiation $†$ & $25(92.6)$ & $276(82.4)$ & 0.17 \\
\hline Temozolomide $†$ & $19(70.4)$ & $243(72.5)$ & 0.81 \\
\hline LOS (days) & & & 0.10 \\
\hline Mean & $8.3 \pm 6.6$ & $6.3(6.3)$ & \\
\hline Median & 6 & 4 & \\
\hline IQR & $3-12$ & $3-8$ & \\
\hline Discharge disposition & & & $<0.0001$ \\
\hline Home & $9(33.3)$ & $254(76.7)$ & \\
\hline Rehab/transfer/died & $18(66.7)$ & $77(23.3)$ & \\
\hline Missing & $0(0)$ & $4(1.2)$ & \\
\hline
\end{tabular}

EOR = extent of resection; IQR = interquartile range; $P R=$ partial resection; $N$ / GTR $=$ near- or gross-total resection; rehab $=$ rehabilitation.

* Values inside parentheses are percentages; means are given with SDs.

$\dagger$ Initial treatment.

\section{Predictors of Readmission}

Higher KPS score (HR 0.96, p = 0.04) and home discharge disposition (HR 0.21, p = 0.003) at index hospitalization were associated with a significantly lower risk of readmission within 30 days; a single-level improvement in functional independence (a 10-point increase in KPS score) reduced the risk of readmission by $4 \%$, while being discharged to home as opposed to being discharged to a rehabilitation facility or being transferred had a 79\% reduction of the hazard of readmission (Table 3 ).

\section{Readmission Cohort}

Among the 27 patients $(7.5 \%)$ who were readmitted within 30 days of initial surgery, $6(22.2 \%)$ were readmitted by Day 7, 14 (51.9\%) by Day 14, and 20 (74.1\%) by Day 21. The average time to readmission was 15.3 days (median 14 days). The average LOS during the index hospitalization was 8.3 days, and the average LOS upon readmission was 7.0 days. The most frequent causes of readmission were neurological complications (37.0\%), followed by infectious complications (29.6\%) and thromboembolic events $(22.2 \%)$. Of the 10 patients readmitted for neurological complications, 4 were readmitted for hydrocephalus/fluid collection, 2 for seizure, 1 for leptomeningeal disease, 1 for hyponatremia, and 1 for hemorrhage (intratumoral). Of the 8 patients readmitted for infectious reasons, 3 were readmitted for surgical site wound infection (SSI), 2 for urinary tract infection (UTI), 2 for neutropenic fever, and 1 for leukocytosis. Finally, of the 6 patients readmitted for thromboembolic events, 5 were admitted for PE and 1 was admitted for DVT (Table 4).

Upon close review of the readmitted cohort, it was observed that 6 patients $(22.2 \%)$ died within 3 months of their index surgery; 2 of these patients had been readmitted for pulmonary embolism (thromboembolic), 2 for infection (including a UTI and an SSI; infectious), 1 for back pain (musculoskeletal), and 1 for hematuria secondary to Foley catheter-induced trauma (other). Five (83.3\%) of these 6 patients were discharged to an acute physical rehabilitation facility after their index hospitalization. The median time in days to readmission for these 6 patients was longer than that for patients surviving more than 3 months (21 vs $13, \mathrm{p}=0.24)$.

\section{Preventable and Surgery-Related Readmissions}

Reasons for readmissions in this cohort were carefully analyzed based on the previously described criteria to determine whether each readmission was preventable or non-preventable. Among the 27 readmissions in which patients returned to the hospital within 30 days of surgery, 19 (70.4\%) were classified as preventable. The majority of these readmissions classified as preventable were for thromboembolic, neurological, or infectious reasons. Six patients were readmitted for venous thromboembolism (VTE), 3 for SSIs, 2 for a UTI, 2 for subgaleal/extraaxial fluid collection, 1 for leukocytosis, 1 for seizure (due to noncompliance with medication), 1 for intratumoral hemorrhage, 1 for back pain, 1 for hyponatremia, and 1 for hematuria second to Foley catheter-induced trauma. The reasons for readmission in the remaining 8 cases (29.6\%) were classified as non-preventable and included disease progression and side effects of treatment (radiotherapy, chemotherapy). More specifically, non-preventable reasons for readmission included 2 cases of neutropenic fever (classified as non-preventable because it is a complication of chemotherapy), 1 case of cerebral edema (second to radiation therapy), 1 patient experiencing seizure (classified 


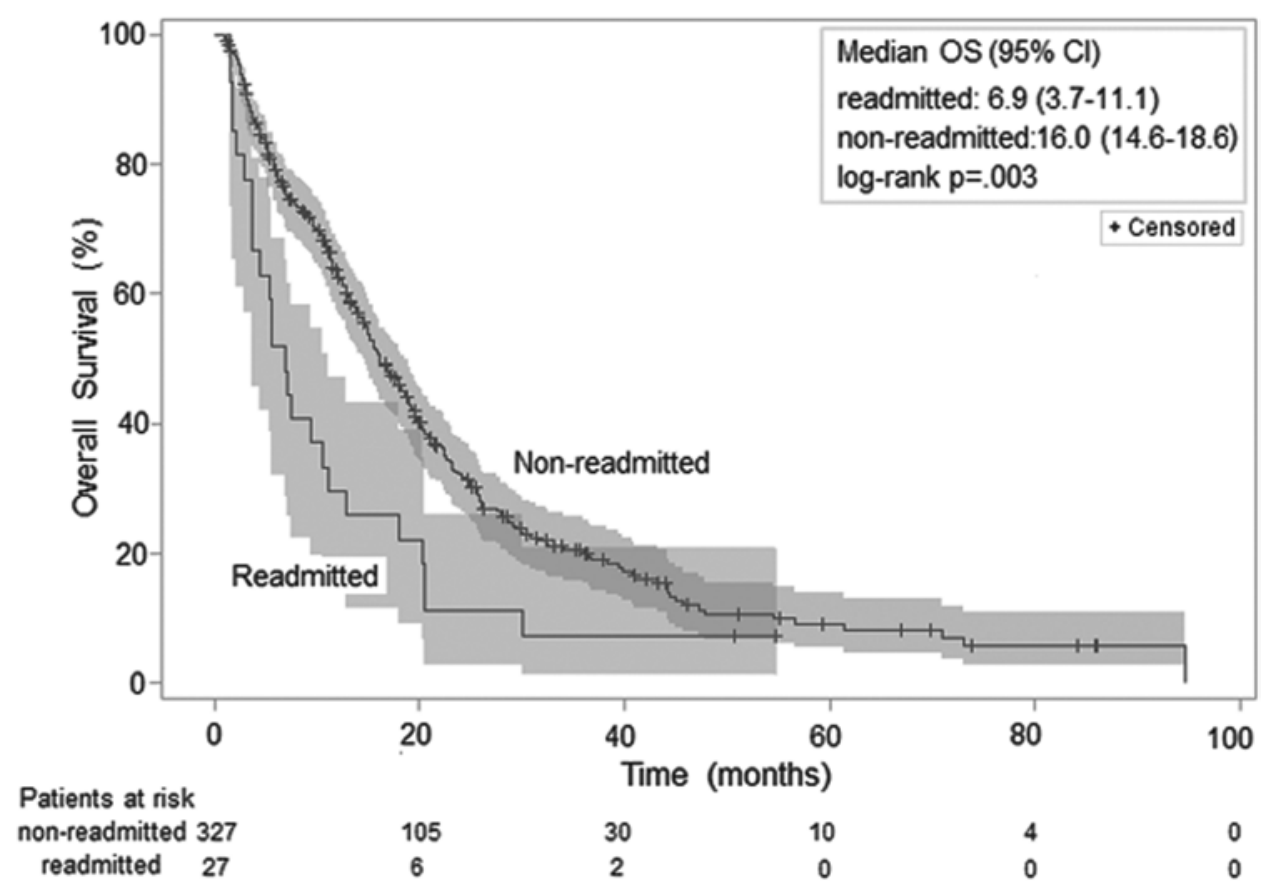

FIG. 1. Overall survival (OS) functions for 327 non-readmitted and 27 readmitted patients within 30 days of the index hospitalization. Kaplan-Meier estimates for overall survival times in months for non-readmitted and readmitted patients.

as non-preventable because the patient was compliant with the prophylactic medication regimen), 1 instance of chest pain (second to coronary artery disease and bypass graft), and 2 for hydrocephalus. Readmissions were further described in terms of whether they were related to either the tumor or treatment of the tumor. For instance, a patient with preexisting coronary artery disease returning to the hospital after a craniotomy with cardiac complications would be considered to have an unrelated readmission. Two (11.1\%) of the 27 readmitted patients were readmitted for reasons unrelated to the disease or treatment. One patient presented with chest pain secondary to coronary artery disease (and was listed as having a cardiac com-

TABLE 2. Adjusted hazard ratio for mortality, $95 \%$ confidence interval, and $p$ values for 354 patients with newly diagnosed glioblastoma*

\begin{tabular}{lcc}
\hline \multicolumn{1}{c}{ Variable } & $\mathrm{HR}(95 \% \mathrm{Cl})$ & $\mathrm{p} \mathrm{Value}$ \\
\hline Readmission & $2.03(1.31-3.12)$ & 0.001 \\
\hline Age, yr increment & $1.03(1.02-1.04)$ & $<0.0001$ \\
\hline KPS, level increment & $0.85(0.78-0.94)$ & 0.001 \\
\hline EOR (ref: biopsy) & & \\
\hline PR & $0.62(0.44-0.87)$ & 0.007 \\
\hline N/GTR & $0.51(0.39-0.69)$ & $<0.0001$ \\
\hline Radiation $\dagger$ & $0.89(0.57-1.40)$ & 0.62 \\
\hline Temozolomide $\dagger$ & $0.44(0.30-0.66)$ & $<0.0001$ \\
\hline SOI & $1.21(1.02-1.44)$ & 0.03 \\
\hline
\end{tabular}

ref $=$ reference.

* Eight patients of the cohort of 362 were excluded from survival analysis since they died during hospitalization or were discharged to hospice. † Initial treatment. plication in Table 4), and another patient (with a known history of chronic back pain and scoliosis) was readmitted for back pain (listed as a musculoskeletal complication in Table 4).

\section{Discussion}

In 2004, the Centers for Medicare and Medicaid Services reported that unplanned hospital readmissions resulted in $\$ 17.4$ billion in costs to Medicare alone. ${ }^{26}$ As a result, in October of 2012 Medicare instituted a 1\% payment penalty for hospitals with excessive early readmissions, as high readmission rates had emerged as indicators

TABLE 3. Adjusted odds ratio for readmission, $95 \%$ confidence interval, and $\mathrm{p}$ values for 354 patients with newly diagnosed glioblastoma*

\begin{tabular}{llc}
\hline \multicolumn{1}{c}{ Variable } & OR $(95 \% \mathrm{Cl})$ & $\mathrm{p} \mathrm{Value}$ \\
\hline Race (ref: non-white) & & 0.21 \\
\hline$\quad$ White & $0.53(0.20-1.43)$ & \\
\hline Radiation (ref: no radiation) & $2.33(0.50-10.8)$ & 0.28 \\
\hline$\quad$ Radiation & & 0.93 \\
\hline Admission type (ref: non-routine) & $0.95(0.35-2.62)$ & \\
\hline$\quad$ Routine & & 0.26 \\
\hline LOS, day increment & $0.96(0.93-1.0)$ & 0.04 \\
\hline KPS, level increment & & 0.003 \\
\hline Discharge disposition (ref: rehab/transfer) & $0.21(0.08-0.59)$ & \\
\hline Home &
\end{tabular}

* Factors significant at the 0.25 or lower level in the univariate setting were adjusted for in the multivariate model. 
TABLE 4. Characteristics of 27 patients readmitted within 30 days of surgery*

\begin{tabular}{|c|c|c|c|c|c|c|c|c|c|}
\hline Case No. & $\begin{array}{c}\text { Age } \\
\text { (yrs), Sex }\end{array}$ & KPS Score & $\begin{array}{c}\text { EOR at } \\
\text { Index Op }\end{array}$ & $\begin{array}{c}\text { LOS at Index } \\
\text { Admission }\end{array}$ & $\begin{array}{l}\text { Discharge } \\
\text { Disposition }\end{array}$ & $\begin{array}{l}\text { Days to } \\
\text { Readmission }\end{array}$ & $\begin{array}{l}\text { LOS at } \\
\text { Readmission }\end{array}$ & $\begin{array}{l}\text { Survival in } \\
\text { Months } †\end{array}$ & Reason for Readmission $\ddagger$ \\
\hline 1 & $75, M$ & 70 & N/GTR & 3 & Transfer & 5 & 3 & 3.7 & Cardiac (P) \\
\hline 2 & $60, M$ & 70 & Biopsy & 1 & Home & 20 & 7 & 11.1 & Neurological (R) \\
\hline 3 & $89, F$ & 70 & PR & 20 & Rehab & 25 & 13 & 17.9 & Infectious (R) \\
\hline 4 & $80, F$ & 40 & PR & 6 & Rehab & 29 & 15 & $1.8(0.7)$ & Infectious $(\mathrm{R}, \mathrm{P})$ \\
\hline 5 & $61, \mathrm{M}$ & 80 & PR & 2 & Home & 30 & 2 & 20.2 & Infectious \\
\hline 6 & $44, \mathrm{M}$ & 100 & PR & 1 & Home & 14 & 3 & $54.7 \S$ & Infectious $(R, P)$ \\
\hline 7 & $69, \mathrm{~F}$ & 90 & PR & 4 & Home & 12 & 5 & 5.4 & Infectious (P) \\
\hline 8 & $46, \mathrm{M}$ & 80 & Biopsy & 6 & Home & 10 & 5 & 10.6 & Neurological \\
\hline 9 & $52, F$ & 60 & PR & 20 & Rehab & 22 & 5 & $1.5(0.2)$ & Infectious $(R, P)$ \\
\hline 17 & $33, \mathrm{M}$ & 90 & N/GTR & 7 & Home & 29 & 6 & $50.7 \S$ & Infectious $(\mathrm{R}, \mathrm{P})$ \\
\hline 10 & $75, \mathrm{~F}$ & 50 & PR & 10 & Rehab & 17 & 4 & 5.5 & Infectious $(R, P)$ \\
\hline 11 & $84, \mathrm{M}$ & 60 & N/GTR & 16 & Rehab & 23 & 28 & $2.1(1.0)$ & Musculoskeletal (P) \\
\hline 12 & $69, \mathrm{M}$ & 60 & N/GTR & 13 & Rehab & 15 & 12 & 6.9 & Neurological $(\mathrm{R}, \mathrm{P})$ \\
\hline 13 & $47, \mathrm{M}$ & 70 & N/GTR & 7 & Rehab & 22 & 6 & 12.8 & Neurological (R) \\
\hline 14 & $67, \mathrm{M}$ & 70 & Biopsy & 1 & Home & 7 & 1 & 3.6 & Neurological $(R, P)$ \\
\hline 15 & $64, \mathrm{M}$ & 70 & Biopsy & 6 & Home & 5 & 6 & 7.1 & Neurological \\
\hline 16 & $61, \mathrm{~F}$ & 40 & Biopsy & 3 & Rehab & 12 & 3 & 4.4 & Neurological (R) \\
\hline 18 & $63, \mathrm{M}$ & 70 & PR & 3 & Rehab & 10 & 4 & 30.0 & Neurological $(R, P)$ \\
\hline 19 & $59, \mathrm{~F}$ & 100 & PR & 2 & Home & 17 & 1 & 7.5 & Neurological $(\mathrm{R}, \mathrm{P})$ \\
\hline 20 & $61, \mathrm{M}$ & 50 & Biopsy & 9 & Rehab & 5 & 9 & 5.5 & Neurological (R) \\
\hline 21 & $65, \mathrm{~F}$ & 70 & PR & 27 & Transfer & 19 & 9 & $1.7(0.3)$ & Other $(R, P)$ \\
\hline 22 & $70, F$ & 50 & PR & 10 & Rehab & 10 & 4 & 2.9 & Thromboembolic (R, P) \\
\hline 23 & $54, \mathrm{M}$ & 50 & Biopsy & 5 & Rehab & 13 & 5 & 9.3 & Thromboembolic (R, P) \\
\hline 24 & $76, F$ & 70 & N/GTR & 12 & Rehab & 9 & 13 & $1.5(1.0)$ & Thromboembolic (R, P) \\
\hline 25 & $52, \mathrm{M}$ & 60 & N/GTR & 6 & Rehab & 7 & 13 & 20.4 & Thromboembolic (R, P) \\
\hline 26 & $34, F$ & 60 & PR & 14 & Rehab & 6 & 5 & 20.6 & Thromboembolic (R, P) \\
\hline 27 & $74, \mathrm{M}$ & 60 & Biopsy & 11 & Rehab & 21 & 4 & 3.7 & Thromboembolic (R, P) \\
\hline
\end{tabular}

* Gray highlighting indicates patients who died within 3 months of their index surgery.

† Values in parentheses indicate duration of survival after readmission.

$\ddagger$ Letters in parentheses indicate complications that were related to surgery $(R)$ and/or preventable $(P)$.

$\S$ Still alive as of data analysis.

of poor quality of delivered care during index hospitalization. . $^{910,19,20,22,34,37}$

Some studies that investigated the correlation between early readmission and overall patient survival concluded that adverse readmissions are associated with shorter patient survival. ${ }^{28,32,37}$ For example, Reddy et al. found that patients readmitted within 30 days of discharge had shorter overall median survival than patients who were not readmitted during that period (11.8 vs 16.5 months, $\mathrm{p}$ $=0.04) .{ }^{28}$ Fewer studies have investigated the association between early readmission and overall patient survival in a surgical cohort. ${ }^{18}$ Our study is the first to consider an internal cohort to investigate the correlation between early readmission and survival in patients with newly diagnosed glioblastoma undergoing a craniotomy. We have shown that an unplanned readmission within 30 days of discharge is associated with a 9-month survival reduction $(\mathrm{p}=0.003)$, consistent with previous studies reporting that readmission within 30 days of surgery is associated with poorer overall survival in hepatopancreatobiliary, thyroid, and pancreatic malignancies..$^{15,28,33,37}$ Furthermore, these findings are supported by the results of our previous nationwide cohort study, which demonstrated that readmissions within 30 days of discharge are associated with a $30 \%$ increase in the hazard of mortality in glioblastoma patients documented in the SEER-Medicare (Surveillance, Epidemiology and End Results) database. ${ }^{26}$

In addition to investigating the association between readmission within 30 days of discharge and survival, we also aimed to elucidate the means by which readmission rates could be reduced. Using an internal database was imperative as this allowed us to carefully review individual medical records for each patient and determine specific reasons for readmission utilizing our preventable versus non-preventable criteria. Although the use of an internal database was advantageous, there are limitations to our study, such as biases of a retrospective study and lack of certain details, including clinician biases and tumor biology. Although factors such as MGMT status, IDHI mutations, and EGFRvIII may well have an impact on survival 
in this patient population, detailed review of our current data showed significant levels of missing data (more than $40 \%$ ). Thus, attempting to draw inferences based on the data from the limited cohort for which complete data are available is likely to reduce the validity of this study, the primary aim of which was to assess the role of readmissions in overall survival. Despite these limitations, this study was unique in its ability to address in detail the course of patients' hospital stays.

As a large portion of the readmitted cohort had preventable readmissions (70.4\%), especially VTE events and SSIs, we further investigated whether appropriate prophylactic measures were taken during index hospitalization. In the 3 cases of SSI, it was determined that all 3 patients were given 2 doses of antibiotics within 24 hours of surgery, indicating that appropriate prophylactic measures were taken. Five of the 6 patients readmitted with a VTE event were given DVT/PE prophylaxis in the form of a compression device, a practice mirrored in the nonreadmitted cohort. None of these patients received chemoprophylactic anticoagulation therapy during their initial admission for surgery, likely due to the increased risk of severe intracranial hemorrhage. ${ }^{34}$ While VTE prophylactic measures have remained unchanged for many years ${ }^{4,35}$ in neurosurgical patients, VTE prophylaxis remains a controversial issue, as the risk-benefit balance is unique for each patient and not clearly defined. Lack of chemoprophylaxis presents a potentially increased risk of postoperative VTE, but administration of anticoagulants such as unfractionated heparin presents the added risk of life-threatening intracranial hemorrhage. ${ }^{6}$

Several of our patients readmitted for preventable reasons had clear inconsistencies in medical care that presumably resulted in their re-hospitalization. For instance, 1 patient was readmitted for hematuria second to Foley catheter-induced trauma, which illustrates a mechanical error resulting in readmission. Furthermore, 1 patient admitted for back pain likely did not have appropriate postdischarge management of pain. The patient readmitted for preventable seizure had seizure due to noncompliance with medication. Additionally, 1 patient with DVT did not receive prophylaxis in the form of a compression device or chemoprophylactic therapy, demonstrating a discrepancy in prophylactic care likely resulting in readmission. The 7 remaining patients of the 19 readmitted for preventable reasons did not have documented discrepancies in their medical care during their index hospitalization to explicitly explain the occurrence of these adverse effects.

Ideally, complications leading to readmission should be prevented to reduce readmissions within the 30 days after discharge, although preventing these readmissions may not alter the course of the disease process. Presentation of the above complications in spite of compliance with accepted standards of care suggests that perhaps current prophylactic standards are inadequate in neurosurgical patients. In orthopedics, it has been suggested that to establish effective SSI prophylaxis, patient-related, surgeryrelated, and infection risk factors should be quantified..$^{16,40}$ Specific measures have also been proposed to prevent the occurrence of UTIs. Several studies have reported that patients often undergo unnecessary catheterization and that catheters are left in place for longer than needed, ${ }^{30}$ and limiting catheter use has been found to be associated with significant decreases in rates of UTI. ${ }^{17,30}$ Use of silver alloy urinary catheters and catheters impregnated with antimicrobial agents have been shown to reduce bacteriuria, ${ }^{7,13,17,30}$ and even simple measures, such as ensuring that the drainage tube sits below the level of the bladder but above the collecting bag, have a significant effect on decreasing the incidence of UTIs. ${ }^{23}$ Therefore, assessment of hazards specific to craniotomy patients may result in increased efficacy in prevention and treatment of these postoperative complications.

In addition to improvements to the in-hospital standard of care, previous studies have also suggested that modifications in care upon discharge and post-discharge followup have the potential to significantly reduce readmission rates postoperatively. Randomized prospective trials have demonstrated that $12 \%$ to $75 \%$ of all readmissions can be prevented simply through patient education, pre-discharge assessment, and adequate domiciliary aftercare. ${ }^{2}$ Readmission rates have been reported to decline after the instatement of pre-discharge reviews and improved post-discharge follow-up care. ${ }^{1,36}$ This may be especially effective in reducing readmissions due to noncompliance with medications post-discharge, a prime example being the patient in our cohort readmitted for seizure due to noncompliance with anticonvulsant medication. These reported studies present a specialized window of opportunity for prevention of readmission: if simple alterations in in-hospital homeostatic care and post-discharge follow-up can help reduce these common complications, reduction in rates of postoperative readmission for patients with newly diagnosed glioblastoma can be achieved on a more definitive basis.

While a primary goal for health care providers is to eliminate unnecessary readmissions, glioblastoma patients present a greater challenge than most medical patients, as the pathophysiology of their disease is unpredictable. ${ }^{34} \mathrm{Al}-$ though SSIs and VTEs, are considered preventable occurrences, the methodology of prevention is more complex in neurosurgical patients, as this cohort is inherently more prone to these complications. ${ }^{14,21,27,39}$ With respect to VTE, tumor biology, ${ }^{31}$ operation time,,$^{24}$ and inability to administer prophylactic anticoagulation during surgery due to risk of intracranial hemorrhage may sometimes supersede adequate prevention of this complication. ${ }^{8}$

Furthermore, complications that would routinely be managed on an outpatient basis require readmission in this cohort due to the sensitivity of postoperative craniotomy patients. In patients who have recently undergone biopsy or resection of glioblastoma, SSIs require readmission due to the imminent risk of CNS infection if the focal infection were to spread subdurally. ${ }^{34}$ Additionally, complications such as postoperative VTE necessitate careful medical management. All 6 patients readmitted for presentation of VTE were in rehabilitation and had lower perioperative KPS scores than the non-readmitted cohort when their DVT/PE was discovered. In a less sensitive cohort, these postoperative events could likely be managed within the rehabilitation facility on an outpatient basis without readmission to a hospital. However, 
postoperative neurosurgical patients require inpatient readmission as a result of the inability to administer a therapeutic level of anticoagulation within 30 days after craniotomy due to the risk of intracranial hemorrhage. ${ }^{34}$ Several studies involving large cohorts of neurosurgical patients have consistently demonstrated a non-zero rate of VTE complications in neurosurgical patients, implying that these adverse events are not entirely preventable in this cohort. ${ }^{5,11,12}$

While some of these complications may not be completely preventable in this patient population due to pathophysiology and preexisting conditions, some complications resulting in adverse readmissions have the potential to be reduced by modification of current practices. Examples include previously mentioned prophylactic measures for UTI as well as improvements in post-discharge patient education and follow-up care. For complications in which alterations in the standard of care may reduce occurrence and thus the resulting readmissions, an effort should be made to universalize these practices. While a reduction in early readmissions may not necessarily result in increased overall survival in this cohort, it is still important to reduce instances of readmission, as this has the potential to relieve some cost and resource burden of this particular patient cohort on the health care system as well as improve patient safety and satisfaction. ${ }^{34}$

\section{Conclusions}

In patients with newly diagnosed glioblastoma, re-hospitalization within 30 days of index surgery was associated with a 9-month reduction in survival. Infectious, neurological, and thromboembolic complications were the leading reasons for readmission. Detailed patient chart reviews demonstrated that $70 \%$ of readmissions were preventable. While some of these complications are inherently not entirely preventable in this cohort, simple improvements in care during hospitalization and after discharge have the potential to reduce the overall occurrence of early readmissions.

\section{References}

1. Bean $P$, Waldron $K$ : Readmission study leads to continuum of care. Nurs Manage 26:65, 67-68, 1995

2. Benbassat J, Taragin M: Hospital readmissions as a measure of quality of health care: advantages and limitations. Arch Intern Med 160:1074-1081, 2000

3. Carapella CM, Telera S, Oppido PA: Surgery of malignant gliomas: advances and perspectives. Curr Opin Oncol 23:624-629, 2011

4. Cerrato D, Ariano C, Fiacchino F: Deep vein thrombosis and low-dose heparin prophylaxis in neurosurgical patients. J Neurosurg 49:378-381, 1978

5. Cifu DX, Kaelin DL, Wall BE: Deep venous thrombosis: incidence on admission to a brain injury rehabilitation program. Arch Phys Med Rehabil 77:1182-1185, 1996

6. Danish SF, Burnett MG, Ong JG, Sonnad SS, MaloneyWilensky E, Stein SC: Prophylaxis for deep venous thrombosis in craniotomy patients: a decision analysis. Neurosurgery 56:1286-1294, 2005

7. Darouiche RO, Smith JA Jr, Hanna H, Dhabuwala CB, Steiner MS, Babaian RJ, et al: Efficacy of antimicrobial-impregnated bladder catheters in reducing catheter-associated bacteriuria: a prospective, randomized, multicenter clinical trial. Urology 54:976-981, 1999

8. Dickinson LD, Miller LD, Patel CP, Gupta SK: Enoxaparin increases the incidence of postoperative intracranial hemorrhage when initiated preoperatively for deep venous thrombosis prophylaxis in patients with brain tumors. Neurosurgery 43:1074-1081, 1998

9. Dignan FL, Potter MN, Ethell ME, Taylor M, Lewis L, Brennan J, et al: High readmission rates are associated with a significant economic burden and poor outcome in patients with grade III/IV acute GvHD. Clin Transplant 27:E56-E63, 2013

10. Friedman B, Basu J: The rate and cost of hospital readmissions for preventable conditions. Med Care Res Rev 61:225240, 2004

11. Frim DM, Barker FG II, Poletti CE, Hamilton AJ: Postoperative low-dose heparin decreases thromboembolic complications in neurosurgical patients. Neurosurgery 30:830-833, 1992

12. Geerts WH, Code KI, Jay RM, Chen E, Szalai JP: A prospective study of venous thromboembolism after major trauma. $\mathbf{N}$ Engl J Med 331:1601-1606, 1994

13. Gentry H, Cope S: Using silver to reduce catheter-associated urinary tract infections. Nurs Stand 19:51-54, 2005

14. Goldfield NI, McCullough EC, Hughes JS, Tang AM, Eastman B, Rawlins LK, et al: Identifying potentially preventable readmissions. Health Care Financ Rev 30:75-91, 2008

15. Greenblatt DY, Weber SM, O'Connor ES, LoConte NK, Liou JI, Smith MA: Readmission after colectomy for cancer predicts one-year mortality. Ann Surg 251:659-669, 2010

16. Horan TC, Gaynes RP, Martone WJ, Jarvis WR, Emori TG: CDC definitions of nosocomial surgical site infections, 1992: a modification of CDC definitions of surgical wound infections. Infect Control Hosp Epidemiol 13:606-608, 1992

17. Huang WC, Wann SR, Lin SL, Kunin CM, Kung MH, Lin $\mathrm{CH}$, et al: Catheter-associated urinary tract infections in intensive care units can be reduced by prompting physicians to remove unnecessary catheters. Infect Control Hosp Epidemiol 25:974-978, 2004

18. Ingraham AM, Cohen ME, Bilimoria KY, Dimick JB, Richards KE, Raval MV, et al: Association of surgical care improvement project infection-related process measure compliance with risk-adjusted outcomes: implications for quality measurement. J Am Coll Surg 211:705-714, 2010

19. Jencks SF, Williams MV, Coleman EA: Rehospitalizations among patients in the Medicare fee-for-service program. $\mathbf{N}$ Engl J Med 360:1418-1428, 2009

20. Kent TS, Sachs TE, Callery MP, Vollmer CM Jr: Readmission after major pancreatic resection: a necessary evil? J Am Coll Surg 213:515-523, 2011

21. Kim H, Ross JS, Melkus GD, Zhao Z, Boockvar K: Scheduled and unscheduled hospital readmissions among patients with diabetes. Am J Manag Care 16:760-767, 2010

22. Lawson EH, Hall BL, Louie R, Ettner SL, Zingmond DS, Han L, et al: Association between occurrence of a postoperative complication and readmission: implications for quality improvement and cost savings. Ann Surg 258:10-18, 2013

23. Maki DG, Tambyah PA: Engineering out the risk for infection with urinary catheters. Emerg Infect Dis 7:342-347, 2001

24. Marras LC, Geerts WH, Perry JR: The risk of venous thromboembolism is increased throughout the course of malignant glioma: an evidence-based review. Cancer 89:640-646, 2000

25. Narita Y: Drug review: Safety and efficacy of bevacizumab for glioblastoma and other brain tumors. Jpn J Clin Oncol 43:587-595, 2013

26. Nuño M, Ly D, Ortega A, Sarmiento JM, Mukherjee D, Black KL, et al: Does 30-day readmission affect long-term 
outcome among glioblastoma patients? Neurosurgery 74:196-204, 2014

27. Rathore SS, Masoudi FA, Wang Y, Curtis JP, Foody JM, Havranek EP, et al: Socioeconomic status, treatment, and outcomes among elderly patients hospitalized with heart failure: findings from the National Heart Failure Project. Am Heart J 152:371-378, 2006

28. Reddy DM, Townsend CM Jr, Kuo YF, Freeman JL, Goodwin JS, Riall TS: Readmission after pancreatectomy for pancreatic cancer in Medicare patients. J Gastrointest Surg 13:1963-1975, 2009

29. Rosenbaum S: The Patient Protection and Affordable Care Act: implications for public health policy and practice. Public Health Rep 126:130-135, 2011

30. Saint S, Wiese J, Amory JK, Bernstein ML, Patel UD, Zemencuk JK, et al: Are physicians aware of which of their patients have indwelling urinary catheters? Am J Med 109:476-480, 2000

31. Sawaya R, Zuccarello M, Elkalliny M, Nishiyama H: Postoperative venous thromboembolism and brain tumors: Part I. Clinical profile. J Neurooncol 14:119-125, 1992

32. Schneider EB, Hyder O, Brooke BS, Efron J, Cameron JL, Edil BH, et al: Patient readmission and mortality after colorectal surgery for colon cancer: impact of length of stay relative to other clinical factors. J Am Coll Surg 214:390399, 2012

33. Schneider EB, Hyder O, Wolfgang CL, Hirose K, Choti MA, Makary MA, et al: Patient readmission and mortality after surgery for hepato-pancreato-biliary malignancies. J Am Coll Surg 215:607-615, 2012

34. Shah MN, Stoev IT, Sanford DE, Gao F, Santiago P, Jaques DP, et al: Are readmission rates on a neurosurgical service indicators of quality of care? Clinical article. J Neurosurg 119:1043-1049, 2013

35. Skillman JJ, Collins RE, Coe NP, Goldstein BS, Shapiro RM, Zervas NT, et al: Prevention of deep vein thrombosis in neu- rosurgical patients: a controlled, randomized trial of external pneumatic compression boots. Surgery 83:354-358, 1978

36. Smith CS: The impact of an ambulatory firm system on quality and continuity of care. Med Care 33:221-226, 1995

37. Tuggle CT, Park LS, Roman S, Udelsman R, Sosa JA: Rehospitalization among elderly patients with thyroid cancer after thyroidectomy are prevalent and costly. Ann Surg Oncol 17:2816-2823, 2010

38. Vaduganathan M, Bonow RO, Gheorghiade M: Thirty-day readmissions: the clock is ticking. JAMA 309:345-346, 2013

39. Weeks WB, Lee RE, Wallace AE, West AN, Bagian JP: Do older rural and urban veterans experience different rates of unplanned readmission to VA and non-VA hospitals? J Rural Health 25:62-69, 2009

40. Wilson AP, Hodgson B, Liu M, Plummer D, Taylor I, Roberts $\mathrm{J}$, et al: Reduction in wound infection rates by wound surveillance with postdischarge follow-up and feedback. Br J Surg 93:630-638, 2006

\section{Author Contributions}

Conception and design: Patil. Acquisition of data: Dickinson, Carico. Analysis and interpretation of data: Nuño. Drafting the article: Dickinson, Carico, Nuño. Critically revising the article: Patil, Dickinson, Carico, Nuño, Mukherjee, Ortega. Reviewed submitted version of manuscript: Dickinson, Carico, Nuño, Mukherjee, Ortega, Black. Administrative/technical/material support: Ortega. Study supervision: Patil, Mukherjee, Black.

\section{Correspondence}

Chirag G. Patil, Center for Neurosurgical Outcomes Research, Department of Neurosurgery, Cedars Sinai Medical Center, Advanced Health Sciences Pavilion, 127 S. San Vicente Blvd., Ste. A6600, Los Angeles, CA 90048. email: chiragpatil@gmail. com. 